\title{
Lithium borates from the glass to the melt: a temperature-induced structural transformation viewed from the boron and oxygen atoms
}

Gérald Lelong, ${ }^{\mathrm{a},}{ }^{*}$ Laurent Cormier, ${ }^{\mathrm{a}}$ Louis Hennet, ${ }^{\mathrm{b}}$ Florent Michel, ${ }^{\mathrm{a}}$ Jean-Pascal Rueff, ${ }^{\mathrm{d}, \mathrm{e}}$ James M. Ablett $^{\mathrm{d}}$ and Giulio Monaco ${ }^{\mathrm{c}}$

${ }^{a}$ Institut de Minéralogie, de Physique des Matériaux et Cosmochimie (IMPMC), Sorbonne Universités - UPMC Univ Paris 06, UMR CNRS 7590, Muséum National d'Histoire Naturelle, IRD UMR 206, 4 Place Jussieu, F75005 Paris, France

${ }^{b}$ Conditions Extrêmes et Matériaux: Haute Température et Irradiation, CNRS-UPR 3079, Orléans, France ${ }^{c}$ European Synchrotron Radiation Facility, 6 rue Jules Horowitz, BP 220, 38043 Grenoble Cedex, France ${ }^{d}$ Synchrotron SOLEIL, L'Orme des Merisiers, BP 48, Saint Aubin, 91192 Gif sur Yvette, France ${ }^{e}$ Laboratoire de Chimie Physique - Matière et Rayonnement, Université Pierre et Marie Curie / CNRS-UMR 7614, 75005 Paris, France

Abstract: A multi-edge study of the local structure of lithium borate glasses and melts has been carried out using X-ray Raman scattering (XRS) as a function of temperature. Thanks to a wide range of compositions, from pure $\mathrm{B}_{2} \mathrm{O}_{3}$ up to the metaborate composition, we are able to finely interpret in detail the modifications of the local environment of both the boron and oxygen atoms in terms of boron coordination number, formation of non-bridging oxygens (NBOs) and polymerization's degree of the borate framework as a function of temperature and composition. A temperature induced ${ }^{[4]} \mathrm{B}$ to ${ }^{[3]} \mathrm{B}$ conversion is observed above the glass transition temperature $\left(\mathrm{T}_{\mathrm{g}}\right)$ from the glass to the melt from the triborate composition up to the metaborate composition. Two distinct melt structures are reported: a well polymerized borate network - with few NBOs - below the triborate composition, and a depolymerized borate network above the diborate composition with a rapid increase of the number of NBOs when $\mathrm{Li}_{2} \mathrm{O}$ is added. These two structurally distinct melts allow explaining the two dynamical regimes observed for lithium ion diffusion.

*Corresponding author: gerald.lelong@,sorbonne-universite.fr 


\section{Introduction}

Ionic transport, diffusivity and viscosity are fundamental properties of melts, whose implications are at the origin of very important processes in material and Earth sciences. The modification of glass structure with temperature is therefore of major interest for the understanding of relaxation phenomena, but also to better understand the solid-liquid transition at the local scale. The hightemperature liquid is a well-defined phase in internal thermodynamic equilibrium, with constant and fast exchanges between atomic configurations through structural relaxation and diffusion processes. Unlike alkali silicate glasses whose properties and structure are mainly governed by the proportion of non-bridging oxygen (NBO), the behavior of alkali borate glasses is more complex. ${ }^{1}$ The higher level of intricacy originates from the existence of two distinct coordination states of boron atoms: the planar units $\mathrm{BO}_{3}$ and the negatively charged tetrahedral units $\mathrm{BO}_{4}$, the combination of these two building blocks allowing a wide variety of tessellations of the tridimensional space. ${ }^{2,3}$

Alkali borate glasses show an increasing fragility as the alkali oxide content increases. ${ }^{4-6}$ This fragile behaviour, characteristic of strong glass-formers, indicates a rapid raise in the configurational entropy above the glass transition temperature $\mathrm{Tg}$, contrary to the strong $\mathrm{B}_{2} \mathrm{O}_{3}$ liquid. The link between structural properties and the entropy increase is not well understood, partly due to the lack of data on the structural reorganization when going from the glass to the liquid. At room temperature, the structure of alkali borate glasses is well described in the literature in terms of short- and medium-range order. ${ }^{1,3,7-12} \mathrm{~A}$ temperature induced ${ }^{[4]} \mathrm{B}$ to ${ }^{[3]} \mathrm{B}$ conversion has also been predicted by molecular dynamics and thermodynamic models ${ }^{13,14}$ and evidenced using in situ high-temperature sample environments coupled to Raman spectroscopy, ${ }^{15-17}$ X-ray or neutron diffraction ${ }^{15,18-21}$ or ${ }^{11} \mathrm{~B}$ NMR. ${ }^{22,23}$ However, experimental data of the $\mathrm{Li}_{2} \mathrm{O}-\mathrm{B}_{2} \mathrm{O}_{3}$ system are rather sparse and mostly concern a few compositions in the range of $20-40 \mathrm{~mol} \%$. It is then worthwhile to study the structural properties of a wide range of compositions as a function of temperature from the glass to the melt.

Central to these problems is the bridging or non-bridging role of oxygen, the archetypal ligand in crystalline and glassy oxides. Many techniques have been used to probe the oxygen environment and in the last few years X-ray Raman scattering (XRS) spectroscopy has emerged as a powerful and versatile local probe of the electronic structure of low- $Z$ elements under a wide range of experimental conditions. ${ }^{24-29}$ XRS spectra are sensitive to a multitude of electronic processes related to inelastic scattering and core electron excitations. These latter ones correspond mainly to the absorption edges of the various elements present in the sample and located in the probed energy-loss window. ${ }^{25,30,31}$ As for the conventional X-ray Absorption Near Edge Spectroscopy (XANES), XRS provides the opportunity to measure several absorption edges, such as the B and O K-edges, almost simultaneously, by simply selecting the energy loss range of interest. In the case of lithium borate glasses, the simultaneous measurement of both $\mathrm{B}$ and $\mathrm{O}$ K-edges is a powerful characterization method to get structural information on the local environment of every atom constituting the glassy network.

In this paper, we present a multi-edge study of the local structure of lithium borate glasses as a function of temperature - from room temperature up to $800^{\circ} \mathrm{C}$. To reach the liquid state, an 
aerodynamic levitation device coupled to a laser heating system was inserted into the sample chamber of a XRS spectrometer, allowing to monitor both the boron coordination number and the formation of NBO atoms as a function of temperature and glass composition.

\section{Materials and Methods}

\section{a. Synthesis}

Lithium borate glasses were synthesized by melting weighed mixtures of lithium carbonate $\left(\mathrm{Li}_{2} \mathrm{CO}_{3}-\right.$ $99.99 \%$ - Alfa Aesar) and boric acid $\left(\mathrm{H}_{3} \mathrm{BO}_{3}-99.5 \%\right.$ - Sigma Aldrich) in a $\mathrm{Pt} / \mathrm{Rh}$ crucible at $1000^{\circ} \mathrm{C}$ during 20 minutes. The crucible was then quenched in water. Five different glasses were prepared with the following molar compositions: $x \mathrm{Li}_{2} \mathrm{O}-(1-x) \mathrm{B}_{2} \mathrm{O}_{3}$, where $x=0.5,0.33,0.25,0.1$ and 0 . The five glasses will be denoted as $\mathrm{LB}\left(\mathrm{Li}_{2} \mathrm{O}-\mathrm{B}_{2} \mathrm{O}_{3}\right)$, LB2 $\left(\mathrm{Li}_{2} \mathrm{O}-2 \mathrm{~B}_{2} \mathrm{O}_{3}\right)$, LB3 $\left(\mathrm{Li}_{2} \mathrm{O}-3 \mathrm{~B}_{2} \mathrm{O}_{3}\right), \mathrm{LB} 9\left(\mathrm{Li}_{2} \mathrm{O}-9 \mathrm{~B}_{2} \mathrm{O}_{3}\right)$ and $\mathrm{v}^{-} \mathrm{B}_{2} \mathrm{O}_{3}$, respectively. Their respective glass transition temperatures are equal to $420^{\circ} \mathrm{C}, 500^{\circ} \mathrm{C}$, $487^{\circ} \mathrm{C}, 343^{\circ} \mathrm{C}$ and $260^{\circ} \mathrm{C} .^{32}$

Spheroidal samples were prepared by melting small pieces of the as-synthesized glasses in a levitation apparatus to obtain homogeneous samples of approximately $2 \mathrm{~mm}$ in diameter. The principle of the levitation chamber is described in detail elsewhere. ${ }^{33}$ For this experiment, the spherical sample was placed in the convergent-divergent nozzle that directed an oxygen gas jet supporting the sample. An additional regulated helium flow was also used to reduce the x-ray absorption in the chamber. Then the sample was melted using two $125 \mathrm{~W} \mathrm{CO}_{2}$ laser beams focused onto the sample by means of spherical mirrors. The specimen temperature was measured by an optical pyrometer operating at a wavelength of $1.625 \mu \mathrm{m}$ at the spot on the surface illuminated by the $\mathrm{x}$-ray beam.

\section{b. XRS Spectrometers}

\section{i. ID16 Beamline - ESRF}

The XRS experiments on LB2, LB3 and LB glasses were carried out at the ID16 Inelastic X-ray beamline of the European Synchrotron Radiation Facility (ESRF). ${ }^{34}$ The inelastic X-ray scattering experiments previously possible on port ID16 of the ESRF are now carried out on port ID20. The incident beam was monochromatized with $\mathrm{Si}(111)$ and $\mathrm{Si}(220)$ monochromators and the beam focused to $115(\mathrm{H}) \times 40(\mathrm{~V}) \mu \mathrm{m}^{2}$. The scattered radiation was analyzed with nine bent $\mathrm{Si}(660)$ analyzers $(R=$ $1 \mathrm{~m})$ and detected with a photon-counting MAXIPIX bidimensional pixel detector. ${ }^{35}$ The spectra were collected by scanning the incident energy while keeping the analyzers energy to $9.6885 \mathrm{keV}$, so that the energy transfer was scanned around both the B $K$-edge at $194 \mathrm{eV}$ and the $\mathrm{O} K$-edge at $535 \mathrm{eV}$. The total energy resolution of the setup was $0.7-0.8 \mathrm{eV}$ as measured by fitting a Gaussian profile to the quasi-elastic scattering peak. All the measurements were made at an average scattering angle of $30^{\circ}$ in 28. Under these conditions all 9 analyzers are in a low exchanged momentum q configuration $(\mathrm{q} \sim$ $2.54 \AA^{-1}$ ) and the corresponding spectra can be summed. An accumulation time of at least $2 \mathrm{~h}$ was 
necessary to achieve good statistics. A background subtraction has been achieved and each spectrum has been normalized to the area. The normalization range was taken between 185 and $218 \mathrm{eV}$ for the $\mathrm{B}$ $\mathrm{K}$-edge and between 530 and $555 \mathrm{eV}$ for the $\mathrm{O} \mathrm{K}$-edge.

\section{ii. GALAXIES Beamline - SOLEIL}

The measurements of $\mathrm{v}-\mathrm{B}_{2} \mathrm{O}_{3}$ and LB9 glasses were performed at the GALAXIES beamline at the SOLEIL Synchrotron. ${ }^{36,37}$ The incident energy was monochromatized with a Si(111) double - crystal monochromator and the beam focused to $\sim 100(\mathrm{H}) \times 30(\mathrm{~V}) \mu \mathrm{m}^{2}$. The scattered radiation was analyzed with 4 spherical $\mathrm{Si}(660)$ analyzers $(\mathrm{R}=1 \mathrm{~m})$ and detected with a silicon drift diode (SDD). The spectra were collected by keeping the analyzers fixed at their quasi-backscattering energy of $9.720 \mathrm{keV}$ (Bragg angle $\sim 86^{\circ}$ ) while scanning the incident X-ray energy. For example, the $\mathrm{O} \mathrm{K}$-edge at $535 \mathrm{eV}$ was measured by varying the incident X-ray energy around $10.255 \mathrm{keV}$. The total energy resolution of the setup was $\sim 1.5 \mathrm{eV}$. All the measurements were made at an average scattering angle of $40^{\circ}$ in $2 \theta$. As in the previous case, under these conditions all 4 analyzers are in a low transfer momentum $q$ configuration ( $\mathrm{q} \sim 3.4+0.4 /-0.6 \AA^{-1}$ ) and the corresponding spectra can be summed. An accumulation time of at least $5 \mathrm{~h}$ was necessary to achieve good statistics. After background subtraction each spectrum has been normalized to the area. The normalization range was taken between 185 and $218 \mathrm{eV}$ for the B K-edge and between 530 and $555 \mathrm{eV}$ for the $\mathrm{O} \mathrm{K}$-edge.

\section{Results}

The five glassy samples - accompanied with the L7B3 composition published elsewhere ${ }^{7}$, span an extended concentration range of $\mathrm{Li}_{2} \mathrm{O}$, starting from pure vitreous $\mathrm{v}-\mathrm{B}_{2} \mathrm{O}_{3}$ up to $70 \mathrm{~mol} . \%$ of $\mathrm{Li}_{2} \mathrm{O}$ in the case of L7B3. All the glasses were measured by XRS from room temperature up to $800-900^{\circ} \mathrm{C}$ depending on the $\mathrm{Li}_{2} \mathrm{O}$ content - the different temperature points are shown in Fig. 1. The highest temperature achieved for each composition was decided on the basis of lithium volatilization, which leads to a rapid reduction of the sphere diameter. All the measurements showed here were performed at a constant sphere diameter.

For each temperature, both the B and O K-edges XRS spectra were measured almost simultaneously. (Fig. 2) As previously described, ${ }^{4,73-40}$ the B K-edge spectrum, which consists of an intense $\pi^{*}$ peak at $194 \mathrm{eV}$ and a broader $\sigma^{*}$ peak located around $203 \mathrm{eV}$, is mainly informative of the nature of the boron coordination polyhedron. On the other hand, the O K-edge XRS spectrum, represented by a narrow peak at $536 \mathrm{eV}$ and a broader one around $545 \mathrm{eV}$, is mainly indicative of the connectivity between the boron polyhedra. 


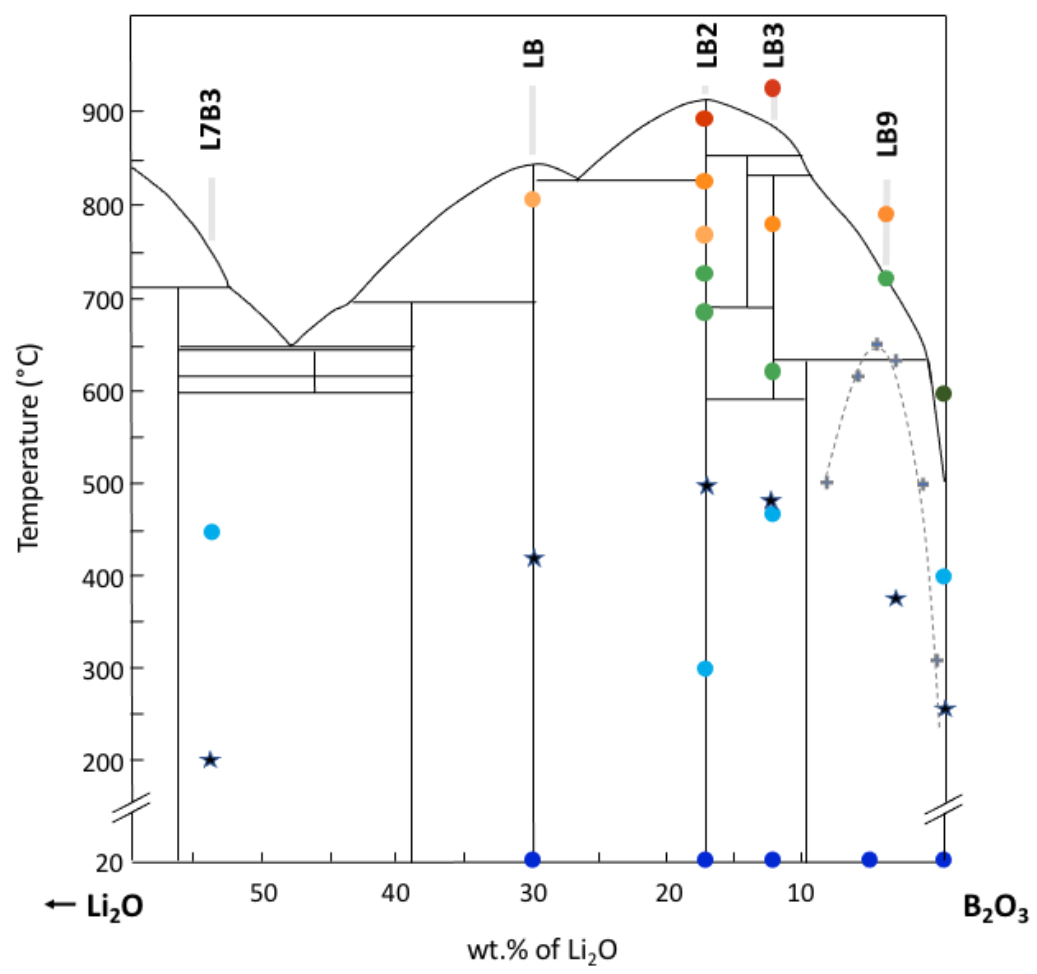

Fig. 1: Phase diagram of the $\mathrm{Li}_{2} \mathrm{O}: \mathrm{B}_{2} \mathrm{O}_{3}$ binary system. The colored filled circles are indicative of the measured temperatures for each glass composition: dark blue (room temperature), cyan $\left(300-500^{\circ} \mathrm{C}\right)$, green $\left(600-700^{\circ} \mathrm{C}\right)$, orange $\left(\sim 800^{\circ} \mathrm{C}\right)$ and red $\left(\sim 900^{\circ} \mathrm{C}\right)$. The L7B3 composition at $450^{\circ} \mathrm{C}$ was measured previously. ${ }^{7}$ The black stars are indicative of the glass transition temperatures. ${ }^{32}$ The dashed grey line shows the subliquidus miscibility dome described by Shaw and Uhlmann ${ }^{41}$

\section{1 Temperature dependence of the B K-edge}

Except for $\mathrm{v}-\mathrm{B}_{2} \mathrm{O}_{3}$ and LB9, all the studied glasses show significant spectral transformations of the $\mathrm{B}$ K-edge as a function of temperature. (Fig. 2) When the temperature rises, the main modifications reside in the general increase in intensity of the $\pi^{*}$ peak and the disappearance of the peak around 200 $\mathrm{eV}$, which are both indicators of a temperature induced ${ }^{[4]} \mathrm{B}$ to ${ }^{[3]} \mathrm{B}$ conversion. This conversion takes place gradually above $T_{g}$ to finally reach some kind of equilibrium around $800-900^{\circ} \mathrm{C}$. Slight fluctuations of the intensity of the $\pi^{*}$ peak caused by subtraction of the Compton scattering background are not significant enough to affect the conclusions. $v-\mathrm{B}_{2} \mathrm{O}_{3}$ and LB9 do not show marked modifications by increasing temperature: only a slight shift of the $\pi^{*}$ peak accompanied by a slight reduction of the intensity is observed with the temperature increase. A similar shift (around $0.1-0.2 \mathrm{eV}$ ) is also observed for the metaborate composition (LB). These subtle modifications are caused by thermal fluctuations similarly to what has been experimentally observed at the Al K-edge of corundum by X-ray absorption near-edge spectroscopy. ${ }^{42}$ 

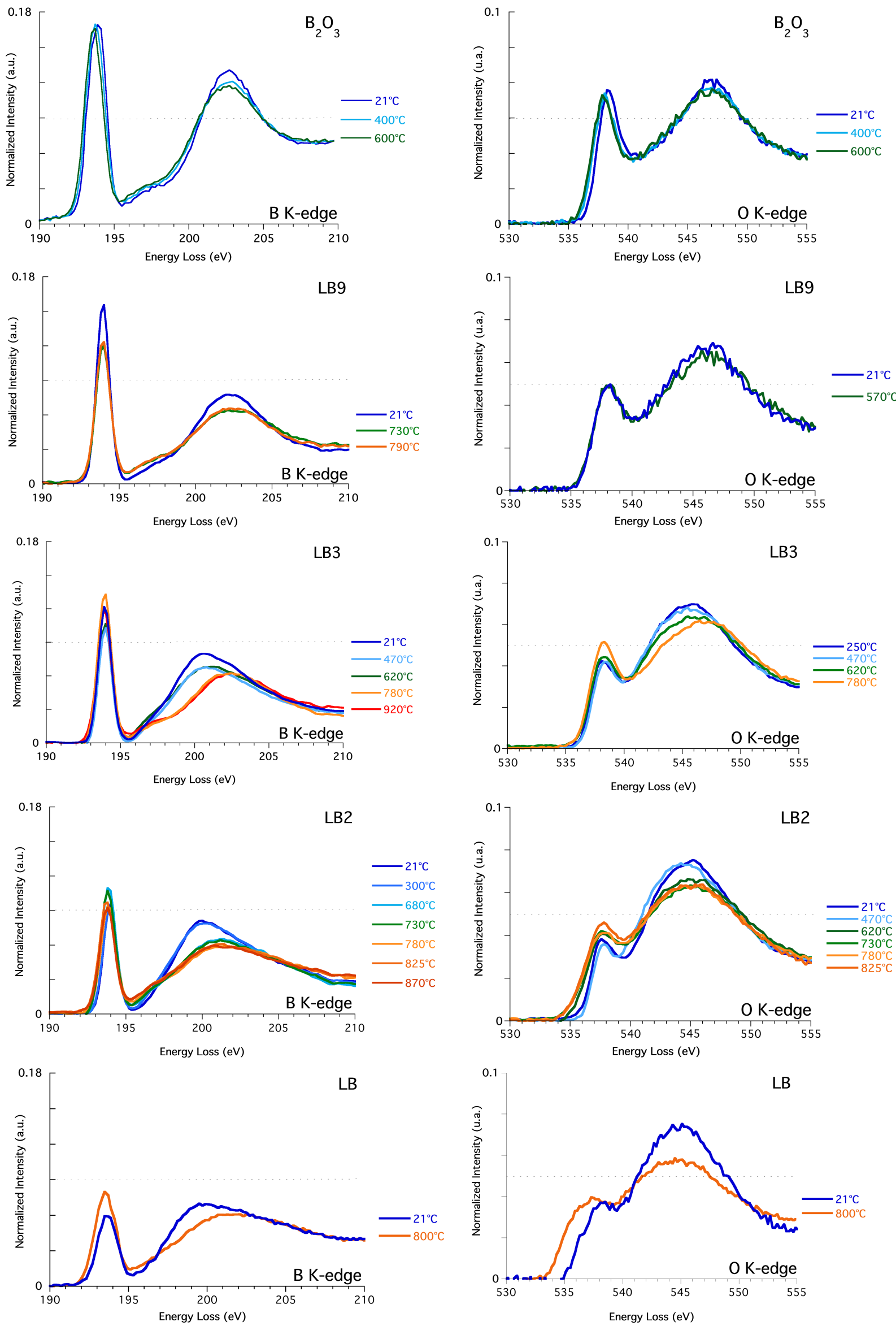

Fig. 2: in situ $B \mathrm{~K}$-edge (left) and $O \mathrm{~K}$-edge (right) $X R S$ spectra of $v-B_{2} O_{3}, L B 9, L B 3, L B 2$ and LB glasses as a function of temperature. All the spectra were measured in the levitation chamber with the exception of the $B K-$ edge of LB9 at room temperature, which has been measured in a standard sample holder. 


\section{2 Temperature dependence of the $\mathrm{O}$ K-edge}

With the exception of a global broadening of the peaks related to an increase of the topological disorder when temperature is increased, the $\mathrm{O}$ K-edge XRS spectra do not show significant changes at low $\mathrm{Li}_{2} \mathrm{O}$ content, i.e. for $\mathrm{v}-\mathrm{B}_{2} \mathrm{O}_{3}$ and LB9 glasses. (Fig. 2) This is in line with the observations for the B K-edge spectra. The O K-edge spectra of LB3 are instead temperature dependent, as shown by the shift of the $\sigma^{*}$ peak at $543 \mathrm{eV}$ and the intensity increase of the $\pi^{*}$ peak around $537 \mathrm{eV}$. From LB3 to $\mathrm{LB}$, the spectral modifications are more and more pronounced, indicative of an increasing ${ }^{[4]} \mathrm{B}$ to ${ }^{[3]} \mathrm{B}$ conversion. ${ }^{38}$ Additionally, an extra peak arises at low energy around $535 \mathrm{eV}$, whose intensity increases with $\mathrm{Li}_{2} \mathrm{O}$ content. This spectral feature is related to the formation of NBOs in the melt as a consequence of the ${ }^{[4]} \mathrm{B}$ to ${ }^{[3]} \mathrm{B}$ conversion. ${ }^{7,38}$

\section{Discussion}

\subsection{Temperature-induced structural modifications on the borate network}

Depending on the stoichiometry, both the short- and medium range orders of alkali borate glasses may be affected by temperature. The monitoring of the proportion of three-fold coordinated boron atoms using XRS is made possible since the $\pi^{*}$ peak of the $\mathrm{B}$ K-edge is solely related to triangular $\mathrm{BO}_{3}$ units. By using a data treatment procedure previously described, ${ }^{7}$ it is possible to follow the proportion of ${ }^{[3]} \mathrm{B}$ as a function of temperature in order to quantify the temperature-induced structural modifications (Fig. 3) ${ }^{15}$ With the exception of LB9 and L7B3, all the glasses show a significant temperature induced ${ }^{[4]} \mathrm{B}$ to ${ }^{[3]} \mathrm{B}$ conversion. The proportion of converted boron atoms is about $15-20 \%$ in the temperature range under study, which is in line with Raman or NMR experimental data on lithium borates. ${ }^{15,16,43,44}$ The average boron coordination number $\mathrm{n}_{\mathrm{BO}}(\mathrm{x}, \mathrm{T})$ is temperature dependent for intermediate compositions, i.e. $\mathrm{LB} 3, \mathrm{LB} 2$ and $\mathrm{LB}$. In the case of $\mathrm{LB} 2, \mathrm{n}_{\mathrm{BO}}(\mathrm{x}, \mathrm{T})$ decreases from 3.5 at $\mathrm{RT}$ to about

3.3-3.4 around $800^{\circ} \mathrm{C}$, i.e. $\frac{\Delta n_{B O}}{\Delta T} \approx-3.10^{-4} K^{-1}$, which agrees with the predictions of thermodynamic models and with the very few neutron diffraction experiments available at elevated temperature. ${ }^{45}$ This decrease of the average B-O coordination number from the glass to the melt takes place only above the glass transition temperature. Below $\mathrm{T}_{\mathrm{g}}$, nothing really happens at the local scale, i.e. some thermal relaxations of defects/constraints on the medium range order may happen without significantly modifying the local boron environment.

Following the conversion reaction $\mathrm{BO}_{4} \Leftrightarrow \mathrm{BO}_{3}+\mathrm{NBO}$, each $\mathrm{BO}_{4}$ unit that is converted into a planar $\mathrm{BO}_{3}$ unit involves the formation of at least one $\mathrm{NBO}$ in order to maintain charge neutrality of the system. Even if the exact quantification of NBOs in borate using XRS is difficult, the intensity of the peak at $535 \mathrm{eV}$ in the $\mathrm{O} \mathrm{K}$-edge spectrum is a good indicator of the concentration of NBOs in the glass. ${ }^{38}$ By plotting the intensity of the peak at $535 \mathrm{eV}$ versus temperature for different $\mathrm{Li}_{2} \mathrm{O}$ concentrations (Fig. 3), we clearly show the correlation between the ${ }^{[4]} \mathrm{B}$ to ${ }^{[3]} \mathrm{B}$ conversion and the formation of NBOs, and that the formation of NBOs is enhanced when the $\mathrm{Li}_{2} \mathrm{O}$ content is increased. NBOs start to appear around $25-30 \mathrm{~mol}^{2} \mathrm{Li}_{2} \mathrm{O}$ and keep increasing up to $50 \mathrm{~mol} \%$. Between 25 and 
$50 \mathrm{~mol}^{2} \mathrm{Li}_{2} \mathrm{O}$, the glass structure is mainly composed by triborate and pentaborate groups, which are gradually replaced by the di-triborate and eventually by the diborate superstructural units both characterized by the presence of a ${ }^{[4]} \mathrm{B}-\mathrm{O}-{ }^{[4]} \mathrm{B}$ bound unit which is energetically less favourable. ${ }^{2,7,44}$ These diborate units seem to be easily breakable upon heating moving on to the formation of $\mathrm{B}_{2} \mathrm{O}^{-}$ and/or $\mathrm{B} \mathrm{O}_{2}{ }^{2-}$ units in the melt, as suggested by MD simulations of Varsamis et al ${ }^{46}$ where $\varnothing$ refers to a bridging oxygen.

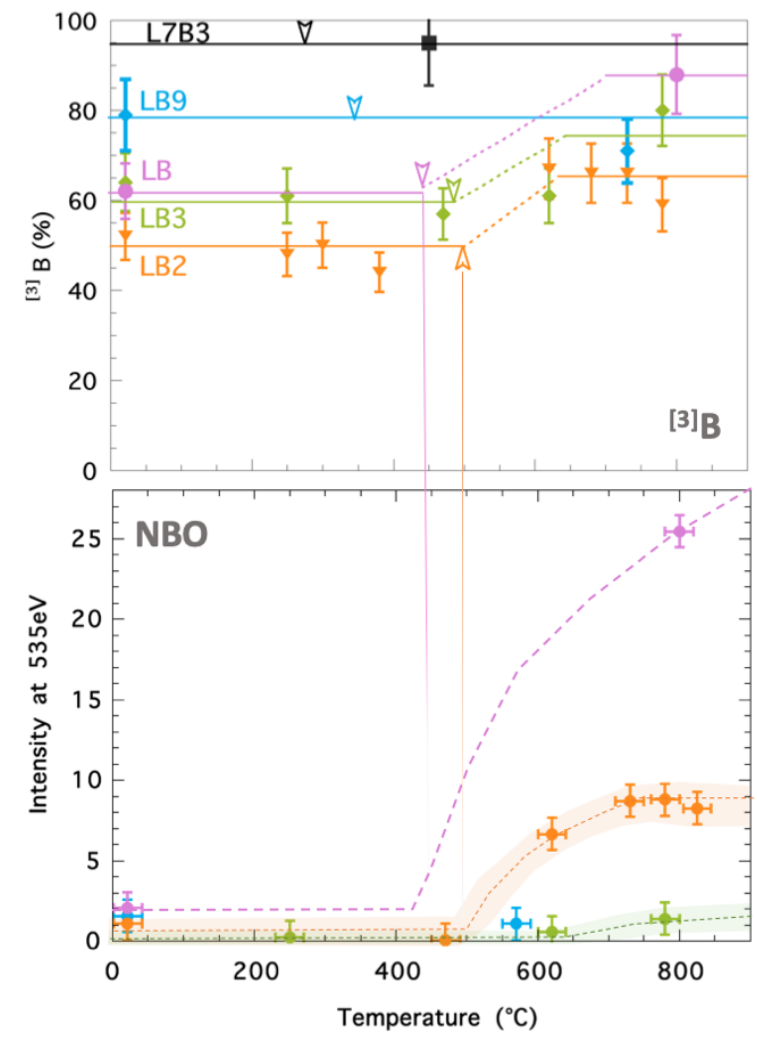

Fig. 3: Evolution of the proportion of ${ }^{[3]} B$ (top) and the intensity at $535 \mathrm{eV}$ (bottom) as a function of temperature and $\mathrm{Li}_{2} \mathrm{O}$ content: LB9 (cyan), LB3 (green), LB2 (orange), LB (pink) and L7B3 from reference ${ }^{7}$ (black). The plain and dashed colored lines are a guide for the eyes, and the arrows are indicative of the glass transition temperatures of the different glasses. ${ }^{13}$

\subsection{Melt structure: existence of two distinct melt structures}

The present study gives access to the structure of the melt for a wide range of $\mathrm{Li}_{2} \mathrm{O}$ concentrations. $\mathrm{By}$ plotting the XRS spectra of both $\mathrm{B}$ and $\mathrm{O}$ K-edges for the five compositions at about $800^{\circ} \mathrm{C}$, it is clear that the melt structure depends on the stoichiometry of the glass (Fig. 4). Two sets of melts compositions can be distinguished:

- a well connected borate network is observed in the melts of $\mathrm{v}^{-} \mathrm{B}_{2} \mathrm{O}_{3}$, LB9 and LB3, characterized by a strong and sharp $\pi^{*}$ peak at the B K-edge and an almost zero intensity at $535 \mathrm{eV}$ at the $\mathrm{O} \mathrm{K}$-edge spectra.

- a more and more depolymerized borate network beyond the LB3 composition with the appearance of numerous NBOs in the melt, as revealed by the intensity increase at $535 \mathrm{eV}$ in the $\mathrm{O}$ K-edge spectra when adding $\mathrm{Li}_{2} \mathrm{O}$. 
These observations indicate that the melt structure goes from a highly polymerized borate network to a more open and depolymerized structure with an increasing $\mathrm{Li}_{2} \mathrm{O}$ content. The transition around 25 $\mathrm{mol}_{\%} \mathrm{Li}_{2} \mathrm{O}$ between these two melt structures has to be put in parallel with the persistence of the boroxol rings in the melt up to $20-25 \mathrm{~mol} \%$, as observed by Raman spectroscopy. ${ }^{2,16,17,47,48}$ The presence of boroxol rings in the liquid state would help to stabilize the connectivity of the borate framework for low-alkali glasses. These two melt structures (polymerized $v$ s. depolymerized) could be at the origin of the subliquidus miscibility dome suggested by Shaw and Uhlmann ${ }^{41}$ and calculated by Kim and Sanders ${ }^{49}$ which extends approximately from $2-18 \mathrm{~mol} \%$ in the case of $\mathrm{Li}_{2} \mathrm{O}-\mathrm{B}_{2} \mathrm{O}_{3}$. For a better insight, the subliquidus miscibility gap has been reproduced in figure 1 . The existence of these two melt structures would explain (i) the non-existence of crystalline phases in this concentration range, and (ii) the coexistence of two sub-domains in the glass with an "alkali-rich" domain and a pure $\mathrm{B}_{2} \mathrm{O}_{3}$ domain containing the boroxol rings. ${ }^{7}$
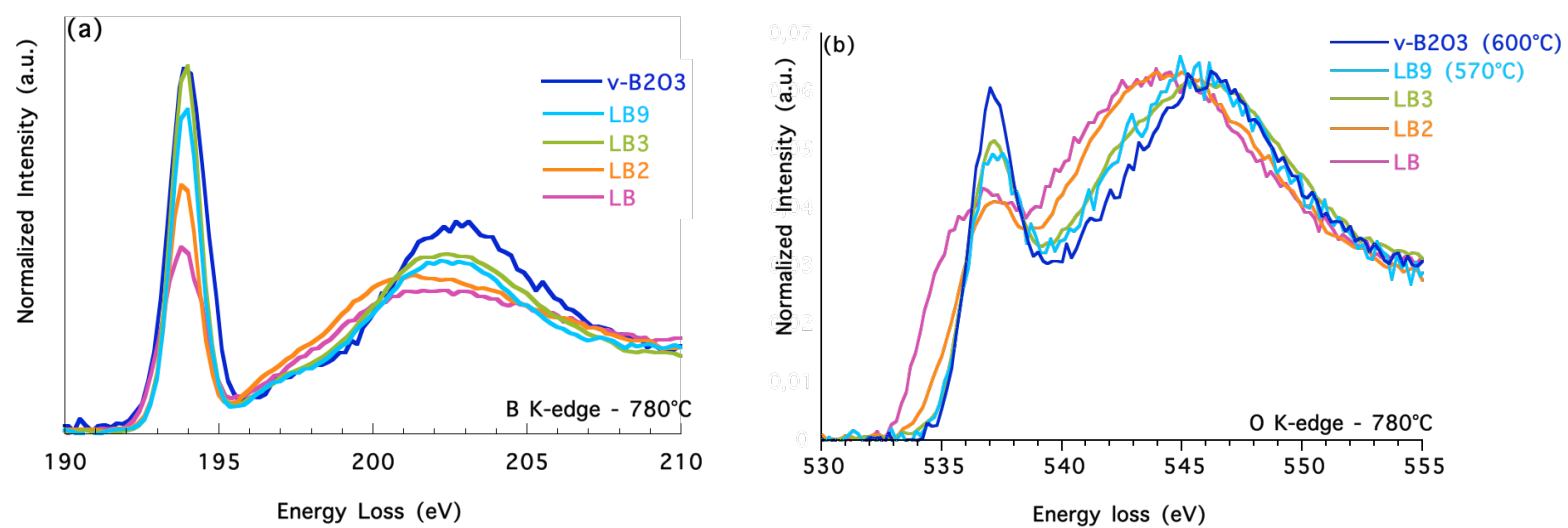

Fig. 4: $B$ K-edge (Left) and $O$ K-edge (Right) XRS spectra of $L B, L B 2$, and LB3 glasses measured at $780^{\circ} \mathrm{C}, L B 9$ at $570^{\circ} \mathrm{C}$ and $v-B_{2} \mathrm{O}_{3}$ at $600^{\circ} \mathrm{C}$.

\subsection{Medium-range order (MRO) in the melt}

Alkali borate glasses are known to show medium range order, which can be classified into welldefined structural motifs known as superstructural units. In order to acquire information about a possible MRO organization in the melt, four crystals with well-defined superstructural units were used as reference samples: $c-\mathrm{Li}_{2} \mathrm{~B}_{4} \mathrm{O}_{7}$ for diborate units $\left(\mathrm{B}_{4} \mathrm{O}_{5} \mathrm{O}_{4}{ }^{2-}\right)$, c- $\mathrm{LiBO}_{2}$ for metaborate chains $\left(\mathrm{BO}_{2}\right)_{\infty}, \mathrm{c}-\mathrm{Li}_{6} \mathrm{~B}_{4} \mathrm{O}_{9}$ for metaborate chains of four-members $\left(\mathrm{B}_{4} \mathrm{O}_{6} \mathrm{Ø}_{3}{ }^{6-}\right)$ and $\mathrm{c}-\mathrm{Li}_{3} \mathrm{BO}_{3}$ for orthoborate units $\left(\mathrm{BO}_{3}{ }^{3-}\right){ }^{2}$ The $\mathrm{O}$ K-edge XRS spectrum of $\mathrm{LB} 2$ at $800^{\circ} \mathrm{C}$ accompanied with those of $\mathrm{c}-\mathrm{Li}_{2} \mathrm{~B}_{4} \mathrm{O}_{7}$ and $\mathrm{c}-\mathrm{LiBO}_{2}$ are shown in Figure 5. Even if the thermal vibrations broaden the spectral features, it is clear that the $\mathrm{O}$ K-edge of the LB2 melt shows similarities with c- $-\mathrm{Li}_{2} \mathrm{~B}_{4} \mathrm{O}_{7}$, particularly when the first $5 \mathrm{eV}$ of the edge are considered. The main difference resides in the formation of a few percent of NBOs which gives an additional contribution at $535 \mathrm{eV}$ and their appearance coincides with the disappearance of some of the $\mathrm{BO}_{4}$ units. This means that the polymerized borate framework is partly preserved in the LB2 melt. On the contrary, the O K-edge of LB melt is intermediate between c$\mathrm{Li}_{6} \mathrm{~B}_{4} \mathrm{O}_{9}$ and $\mathrm{c}-\mathrm{Li}_{3} \mathrm{BO}_{3}$, (Fig. 5) suggesting that the polymerized structure of the LB glass disappears almost completely at high-temperature to finally be constituted by a mixture of metaborate chains of four $\mathrm{BO}_{3}$ members $\left(\mathrm{B}_{4} \mathrm{O}_{6} \varnothing_{3}{ }^{6-}\right)$, orthoborate units $\left(\mathrm{BO}_{3}{ }^{3-}\right)$ and probably pyroborate units $\left(\mathrm{B}_{2} \mathrm{O}_{4}{ }^{4-}\right)$. 
Consequently, the structure of LB melt has to show NBO's rich domains, in which lithium ions are free to move. These two examples highlight the existence of two distinct melt structures up to the diborate composition, i.e. 33 mol\%. Figure 6 schematically summarizes the main temperature induced structural modifications in the $\mathrm{Li}_{2} \mathrm{O}-\mathrm{B}_{2} \mathrm{O}_{3}$ system going from the glass to the melt.
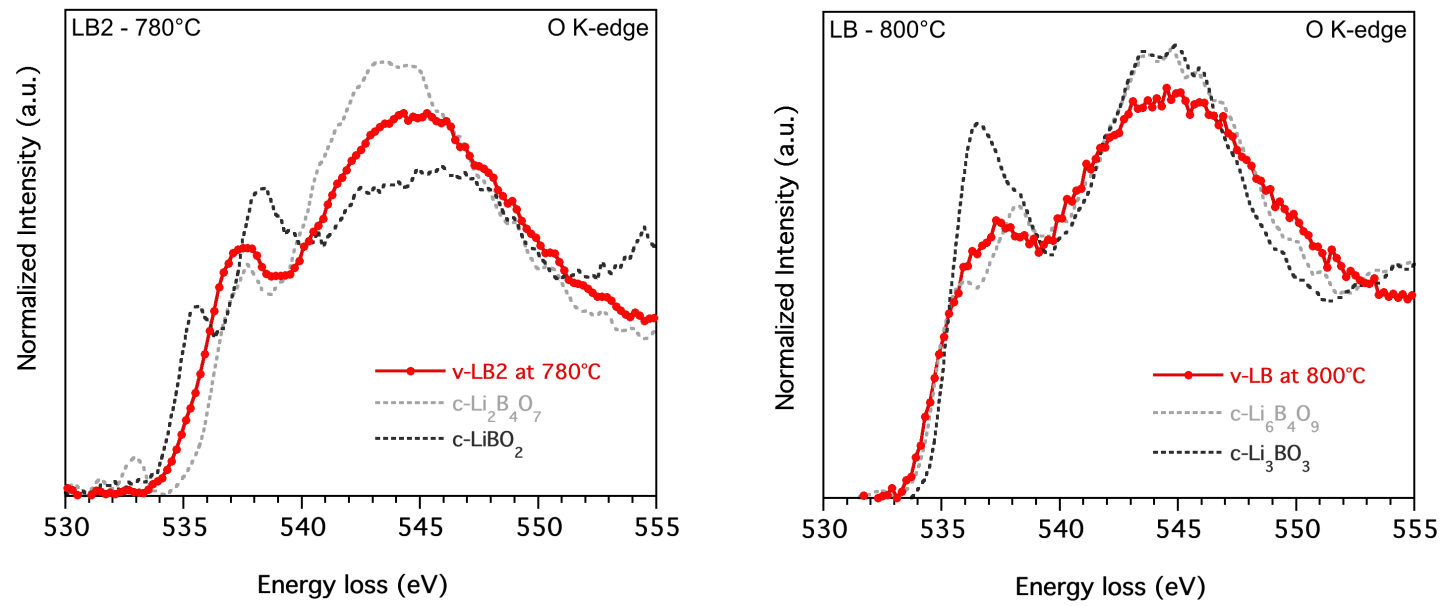

Fig. 5: Left: $O$ K-edge XRS spectra of LB2 melt (red points) accompanied with two crystalline phases: $\mathrm{Li}_{2} \mathrm{~B}_{4} \mathrm{O}_{7}$ (grey) and $\mathrm{LiBO}_{2}$ (black). Right: O K-edge XRS spectra of LB melt (red points) accompanied with two crystalline phases: $\mathrm{Li}_{6} \mathrm{~B}_{4} \mathrm{O}_{9}$ (grey) and $\mathrm{Li}_{3} \mathrm{BO}_{3}$ (black).

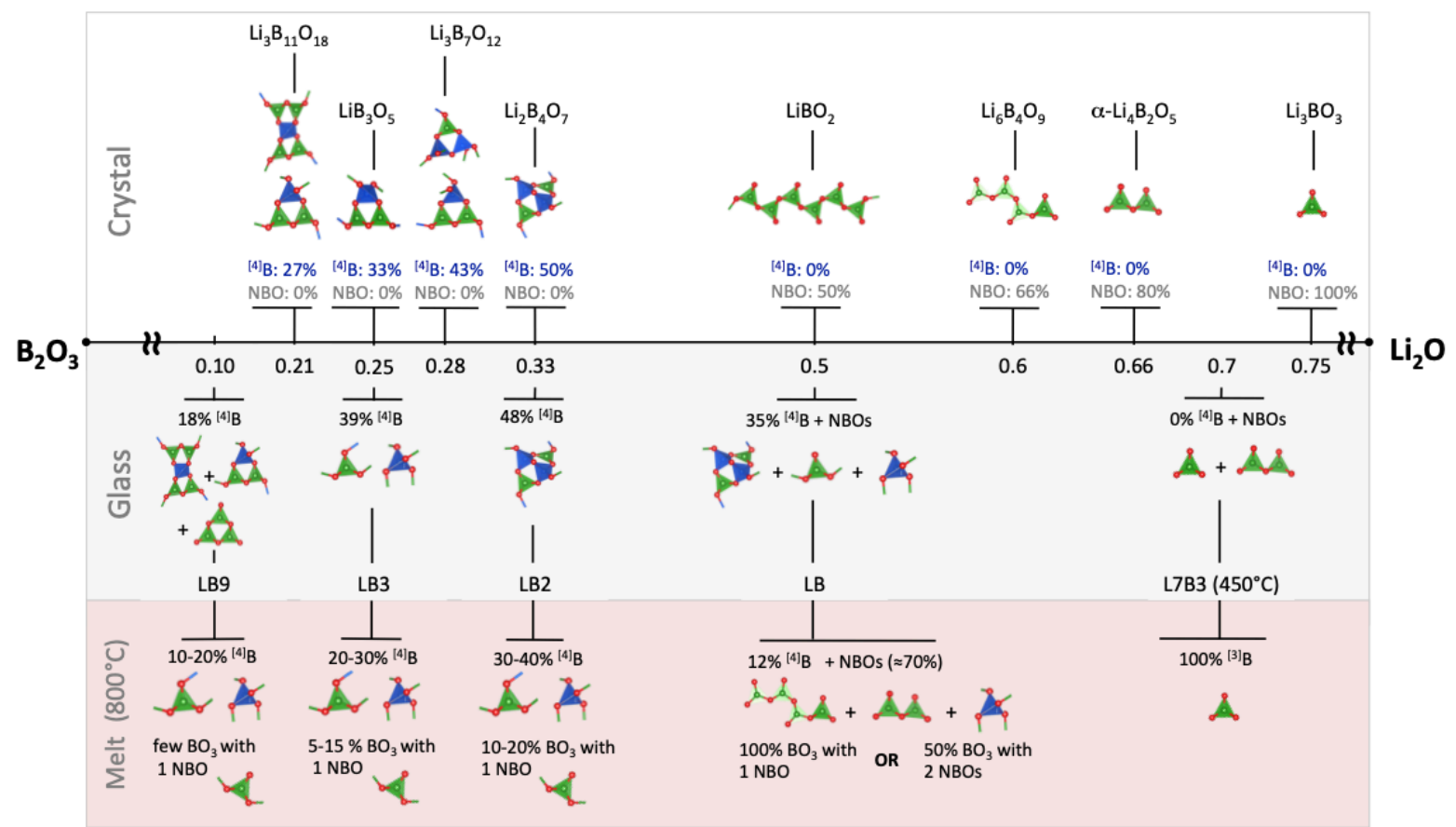

Fig. 6: Summary of the superstructural units found in crystalline phases (top), in glasses at room temperature (grey-shaded area) and in the melt (red-shaded area) for the $\mathrm{Li}_{2} \mathrm{O}-\mathrm{B}_{2} \mathrm{O}_{3}$ system.

\subsection{Melt structure vs density at $800^{\circ} \mathrm{C}$}

Density is related to the atomic spatial distribution, i.e. the stacking of atoms, which will depend on the short-range order, but also on the medium-range organization via the composition in superstructural units. By plotting the structural characteristics of the melt at $800^{\circ} \mathrm{C}$, i.e. the intensity of the peak at $535 \mathrm{eV}$ relative to the presence of NBOs and the proportion of ${ }^{[3]} \mathrm{B}$, and the variation of the 
density at $800^{\circ} \mathrm{C}^{50}$ as a function of $\mathrm{Li}_{2} \mathrm{O}$ content, (Fig. 7) three domains emerge from the composition plot :

Domain $1\left(\mathbf{0 - 3 0} \mathbf{~ m o l \% ~} \mathbf{L i}_{2} \underline{\mathbf{O}}\right)$ : a gradual ${ }^{[3]} \mathrm{B} \rightarrow{ }^{[4]} \mathrm{B}$ conversion is observed in the melt when lithium oxide is added. With the introduction of $\mathrm{Li}^{+}$cations, the electroneutrality is fulfilled by the formation of negatively charged $\left(\mathrm{BO}_{4}\right)^{-}$tetrahedral units - no or very few NBOs are observed in the $0-30 \mathrm{~mol}^{2} \mathrm{Li}_{2} \mathrm{O}$ concentration range. The borate network is fully polymerized on the overall concentration range, but the $3 \mathrm{D}$ connectivity increases with the formation of the $\mathrm{BO}_{4}$ units and the gradual disassembling of the boroxol rings into triborate and then ditriborate units. ${ }^{16}$ As a consequence, the density of the melt increases.

Domain 2 (30-45 $\left.\mathbf{~ m o l \% ~} \mathbf{L i}_{2} \mathbf{O}\right)$ ): between 30 and $45 \mathrm{~mol} \%$, there is a competition between the ${ }^{[3]} \mathrm{B}$ $\rightarrow{ }^{[4]} \mathrm{B}$ conversion and the appearance of NBOs. Both processes have opposite effects on the melt density: the ${ }^{[3]} \mathrm{B} \rightarrow{ }^{[4]} \mathrm{B}$ conversion when $\mathrm{Li}_{2} \mathrm{O}$ is added tends towards a densification of the melt whereas the formation of NBOs leads to a depolymerization of the borate network and consequently to an increase of the melt volume. A change of slop of density at $800^{\circ} \mathrm{C}$ as a function of concentration is observed in this concentration domain, where the vitrification enthalpy reaches its maximum value. ${ }^{51}$

Domain 3 (45-70 mol\% $\mathbf{L i}_{2} \mathbf{2}$ ): from 45 up to $70 \mathrm{~mol} \%$, the NBO content increases drastically supplanting the ${ }^{[3]} \mathrm{B} \rightarrow{ }^{[4]} \mathrm{B}$ conversion. A progressive depolymerization of the borate network is observed leading to a decrease of the melt density.

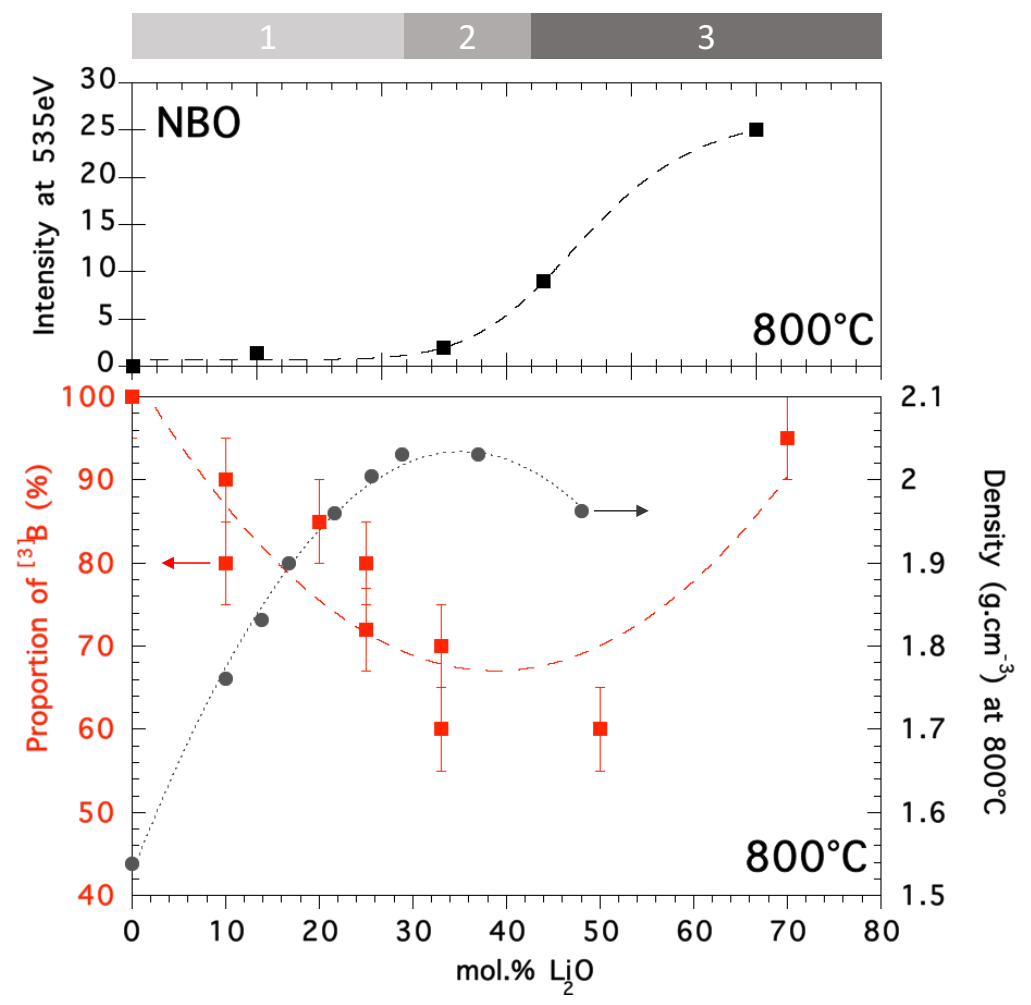


Fig. 7: Evolution of the intensity of the peak at $535 \mathrm{eV}$ related with NBOs (black colored squares), the proportion of ${ }^{[3]} \mathrm{B}$ (red colored squares) and the density ${ }^{50}$ at $800^{\circ} \mathrm{C}$ (grey colored circles) as a function of Li $i_{2} \mathrm{O}$ content. The dashed lines are a guide for the eyes.

\subsection{Melt structure vs Li diffusion}

The structural description of the melt in the section 4.4 is fully corroborated by the constant decrease of the activation energy of lithium diffusion above 20-30 mol\% observed by high-temperature pulsed field gradient NMR. ${ }^{52}$ This decrease is a direct consequence of the reduction of dimensionality of the borate framework with the appearance of NBOs in the melts for $\mathrm{Li}_{2} \mathrm{O}$ content above $45 \mathrm{~mol} \%{ }^{53}$ Both the diffusion coefficient of the lithium ions ${ }^{52}$ and the intensity of the NBO's peak at $535 \mathrm{eV}$ measured at $800^{\circ} \mathrm{C}$ are fully correlated, as shown on the Figure 8 . The disappearance of $\mathrm{BO}_{4}$ units and the formation of NBOs are correlated with a change in the structural role of $\mathrm{Li}$. In the presence of $\mathrm{BO}_{4}, \mathrm{Li}^{+}$ cations play a charge compensating role around the negatively charged $\mathrm{BO}_{4}$ tetrahedra while $\mathrm{Li}^{+}$ cations are modified when associated with NBOs. This change in the Li structural role results in modifications of the local Li environment, as observed by neutron diffraction. ${ }^{11}$

The three structural domains described above are in fair agreement with the dynamical behavior of $\mathrm{Li}$ cations, i.e. a relatively slow translational diffusion up to $30 \mathrm{~mol} \%$ corresponding to almost trapped ions in borate cages, followed by a steadily increase of the translational properties above $30 \mathrm{~mol} \%$. The role of NBOs in the mobility of Li cations is then fully pointed out, and is indicative of the presence of NBO rich-domains, whose size increases from $30 \mathrm{~mol} \%$ up to $50 \mathrm{~mol} \%$, and where $\mathrm{Li}$ ions are free to move. This behavior seems to remain upon quenching, since the ionic conductivity of the glass is enhanced above $30-40 \mathrm{~mol} \%{ }^{54}$

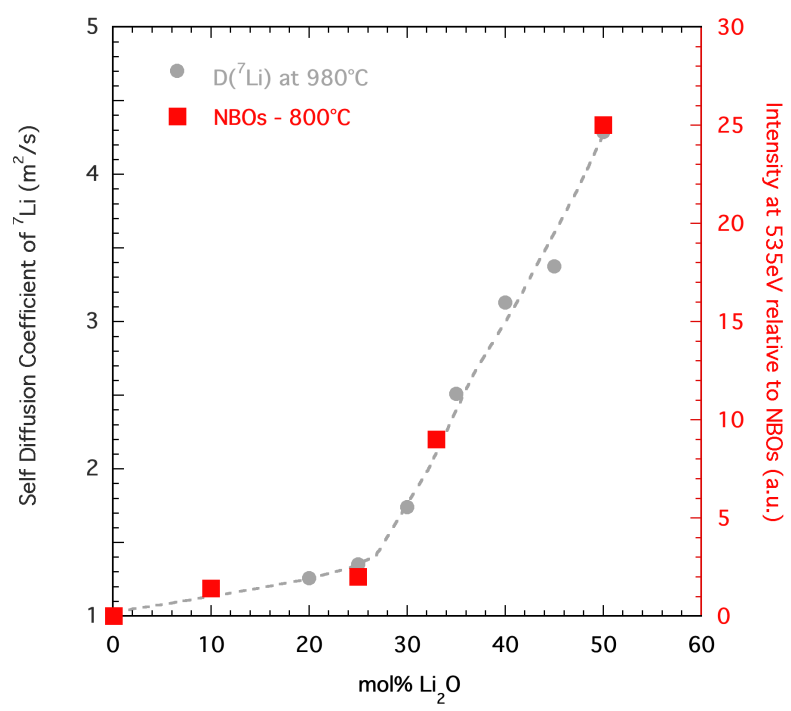

Fig. 8: Comparison between the intensity of the peak at $535 \mathrm{eV}$ at $800^{\circ} \mathrm{C}$, which is indicative of the proportion of $\mathrm{NBOS}$ (red squares) and the self-diffusion coefficient of ${ }^{7}$ Li measured by $N M R^{52}$ at $975^{\circ} \mathrm{C}$ (grey circles) as a function of $\mathrm{Li}_{2} \mathrm{O}$ content.

\section{Conclusions}


This exhaustive study on the temperature behavior of lithium borate glasses and melts allowed us to monitor simultaneously the coordination number of boron atoms and the $\mathrm{BO} / \mathrm{NBO}$ ratio as a function of both temperature and composition. Low lithium content glasses ( $\left(\mathrm{v}-\mathrm{B}_{2} \mathrm{O}_{3}\right.$ and LB9) show almost no modification of their local structure with heating, probably because of the reminiscence of boroxol rings that help to stabilize the melt structure. A temperature induced ${ }^{[4]} \mathrm{B} \rightarrow{ }^{[3]} \mathrm{B}$ conversion is observed above $\mathrm{T}_{\mathrm{g}}$ from the glass to the melt from the triborate composition up to the metaborate composition. The non-monotonic evolution of the melt density of the melt at $800^{\circ} \mathrm{C}$ can be easily explained in terms of ${ }^{[4]} \mathrm{B} \rightarrow{ }^{[3]} \mathrm{B}$ conversion and formation of NBOs.

Two distinct melt structures were evidenced: a well polymerized borate network - with few NBOs below the triborate composition, and a depolymerized borate network above the diborate composition with a rapid increase of the number of NBOs when $\mathrm{Li}_{2} \mathrm{O}$ is added. These two structurally distinct melts allow for explaining the two dynamical regimes observed for lithium ion diffusion, ${ }^{52}$ and could be at the origin of the increasing fragility as the alkali oxide content increases. This study highlights also the major role of oxygen, and especially NBOs in the structural properties of the melt and its consequence on the diffusivity of atoms.

\section{Acknowledgements}

The authors acknowledge the European Synchrotron Radiation Facility and SOLEIL for provision of synchrotron radiation facilities (Proposals HD-490 and 20141349, respectively).

\section{Supporting Information}

XRS data treatment is fully described in the electronic supporting information for both B and O Kedges.

\section{References}

(1) Wright, A. C. Borate versus Silicate Glasses: Why They Are so Different? Phys. Chem. Glas. Eur. J. Glass Sci. Technol. B 2010, 51 (5), 233-265.

(2) Wright, A. C. Borate Structures: Crystalline and Vitreous. Phys. Chem. Glas. Eur. J. Glass Sci. Technol. B 2010, 51 (1), 39.

(3) Wright, A. C. My Borate Life: An Enigmatic Journey. Int. J. Appl. Glass Sci. 2015, 6 (1), $45-63$.

(4) Angell, C. A. Relaxation in Liquids, Polymers and Plastic Crystals - Strong/Fragile Patterns and Problems. J. Non-Cryst. Solids 1991, 131-133, 13-31.

(5) Matsuda, Y.; Fukawa, Y.; Kawashima, M.; Kojima, S.; Tokuyama, M.; Oppenheim, I.; Nishiyama, H. Fragility Variation of Lithium Borate Glasses Studied by Temperature-Modulated DSC; AIP, 2008 ; Vol. 982, pp 207-210.

(6) Mauro, J. C.; Gupta, P. K.; Loucks, R. J. Composition Dependence of Glass Transition Temperature and Fragility. II. A Topological Model of Alkali Borate Liquids. J. Chem. Phys. 2009, 130, 234503.

(7) Lelong, G.; Cormier, L.; Hennet, L.; Michel, F.; Rueff, J.-P.; Ablett, J. M.; Monaco, G. Lithium Borate Crystals and Glasses: How Similar Are They? A Non-Resonant Inelastic X-Ray Scattering Study around the B and O K -Edges. J. Non-Cryst. Solids 2017, 472, 1-8.

(8) Chryssikos, G. D.; Duffy, J. A.; Hutchinson, J. M.; Ingram, M. D.; Kamitsos, E. I.; Pappin, A. J. Lithium Borate Glasses: A Quantitative Study of Strength and Fragility. J. Non-Cryst. Solids 1994, 172-174, $378-383$.

(9) Kamitsos, E. I.; Patsis, A. P.; Karakassides, M. A.; Chryssikos, G. D. Infrared Reflectance Spectra of Lithium Borate Glasses. J. Non-Cryst. Solids 1990, 126 (1-2), 52-67.

(10) Kamitsos, E. I.; Chryssikos, G. D. Borate Glass Structure by Raman and Infrared Spectroscopies. J. Mol. Struct. 1991, 247, 1-16. 
(11) Majérus, O.; Cormier, L.; Calas, G.; Beuneu, B. Structural Modifications between Lithium-Diborate Glasses and Melts: Implications for Transport Properties and Melt Fragility. J. Phys. Chem. B 2003, 107, 13044.

(12) Cormier, L.; Calas, G.; Beuneu, B. Quantification of Boron Coordination Changes between Lithium Borate Glasses and Melts by Neutron Diffraction. Phys Chem Glas. 2009, 50 (3), 195.

(13) Borate Glasses, Crystals \& Melts: [Proceedings of the Second International Conference on Borates Glasses, Crystals and Melt Held at The Cosener's House, Abingdon, UK on 22-25 July 1996]; Wright, A. C., Society of Glass Technology, International Conference on Borates Glasses, Crystals and Melt, Eds.; Society of Glass Technology: Sheffield, 1997.

(14) Varsamis, C.-P. E.; Vegiri, A.; Kamitsos, A. I. A Molecular Dynamics Study of Li-Doped Borate Glasses. Condens. Matter Phys. 2001, 4 (1(25)), 14.

(15) Cormier, L.; Majérus, O.; Neuville, D. R.; Calas, G. Temperature-Induced Structural Modifications Between Alkali Borate Glasses and Melts. J. Am. Ceram. Soc. 2006, 89 (1), 13-19.

(16) Osipov, A. A.; Osipova, L. M. Structure of Lithium Borate Glasses and Melts: Investigation by High Temperature Raman Spectroscopy. Phys. Chem. Glas. Eur. J. Glass Sci. Technol. B 2009, 50 (6), 343354.

(17) Yano, T.; Kobayashi, T.; Shibata, S.; Yamane, M. High Temperature Raman Spectra of $\mathrm{R}_{2} \mathrm{O}_{-} \mathrm{B}_{2} \mathrm{O}_{3}$ Glass Melts (R=Ag, Li, Na and K). Proc. XIX Int. Congr. Glass 2002, 43C, 5.

(18) Alderman, O. L. G.; Liška, M.; Macháček, J.; Benmore, C. J.; Lin, A.; Tamalonis, A.; Weber, J. K. R. Temperature-Driven Structural Transitions in Molten Sodium Borates Na2O-B2O3 : X-Ray Diffraction, Thermodynamic Modeling, and Implications for Topological Constraint Theory. J. Phys. Chem. C 2015, 120, 553-560.

(19) Herms, G.; Sakowsky, J. X ray Diffraction Studies of Structural Changes in Molten Borate Glasses. Phys. Chem. Glas. 2000, 41 (5), 309-312.

(20) Majérus, O.; Cormier, L.; Calas, G.; Beuneu, B. Temperature-Induced Boron Coordination Change in Alkali Borate Glasses and Melts. Phys. Rev. B 2003, 67, 24210.

(21) Alderman, O. Borate Melt Structure: A Short Review. Phys. Chem. Glas. Eur. J. Glass Sci. Technol. Part $B$ 2018, 59 (1), 1-10.

(22) Sen, S.; Xu, Z.; Stebbins, J. F. Temperature Dependent Structural Changes in Borate, Borosilicate and Boroaluminate Liquids: High-Resolution 11B, 29Si and 27Al NMR Studies. J. Non-Cryst. Solids 1998, 226, 29-40.

(23) Sen, S. Temperature Induced Structural Changes and Transport Mechanisms in Borate, Borosilicate, and Boroaluminate Liquids: High-Resolution and High-Temperature NMR Results. J. Non-Cryst. Solids 1999, 253, 84-94.

(24) Lee, S. K.; Eng, P. J.; Mao, H.-K. Probing of Pressure-Induced Bonding Transitions in Crystalline and Amorphous Earth Materials: Insights from X-Ray Raman Scattering at High Pressure. Rev. Mineral. Geochem. 2014, 78, 139-174.

(25) Sternemann, C.; Wilke, M. Spectroscopy of Low and Intermediate Z Elements at Extreme Conditions: In Situ Studies of Earth Materials at Pressure and Temperature via X-Ray Raman Scattering. High Press. Res. 2016, 36 (3), 275-292.

(26) Lee, S. K.; Eng, P. J.; Mao, H.-K.; Meng, Y.; Newville, M.; Hu, M. Y.; Shu, J. Probing of Bonding Changes in B2O3 Glasses at High Pressure with Inelastic X-Ray Scattering. Nat. Mater. 2005, 4, 851854.

(27) Lelong, G.; Cormier, L.; Ferlat, G.; Giordano, V.; Henderson, G. S.; Shukla, A.; Calas, G. Evidence of Fivefold-Coordinated Ge Atoms in Amorphous GeO2 under Pressure Using Inelastic x-Ray Scattering. Phys. Rev. B 2012, 85 (13), 134202.

(28) Petitgirard, S.; Sahle, C. J.; Weis, C.; Gilmore, K.; Spiekermann, G.; Tse, J. S.; Wilke, M.; Cavallari, C.; Cerantola, V.; Sternemann, C. Magma Properties at Deep Earth's Conditions from Electronic Structure of Silica. Geochem. Perspect. Lett. 2019, 9, 32-37.

(29) Lee, S. K.; Kim, Y.-H.; Chow, P.; Xiao, Y.; Ji, C.; Shen, G. Amorphous Boron Oxide at Megabar Pressures via Inelastic X-Ray Scattering. Proc. Natl. Acad. Sci. 2018, 115, 5855-5860

(30) Rueff, J.-P.; Shukla, A. Inelastic X-Ray Scattering by Electronic Excitations under High-Pressure. Rev. Mod. Phys. 2010, 82, 847-896.

(31) Sahle, Ch. J.; Mirone, A.; Niskanen, J.; Inkinen, J.; Krisch, M.; Huotari, S. Planning, Performing and Analyzing X-Ray Raman Scattering Experiments. J. Synchrotron Radiat. 2015, 22 (2), 400-409.

(32) Feller, S. A.; Kottke, J.; Welter, J.; Nijhawan, S.; Boekenhauer, R.; Zhang, H.; Feil, D.; Parameswar, C.; Budhwani, K.; Affatigato, M.; Bhatnagar, A.; Bhasin, G.; Bhowmik, S.; Mackenzie, J.; Royle, M.; Kambeyanda, S.; Pandikuthira, P.; Sharma, M. Physical Properties of Alkali Borosilicates Glasses. In Borate Glasses, Crystals \& Melts: [Proceedings of the Second International Conference on Borates Glasses, Crystals and Melt Held at The Cosener's House, Abingdon, UK on 22-25 July 1996]; Sheffield, 1997.

(33) Hennet, L.; Holland Moritz, D.; Weber, R.; Meyer, A. High-Temperature Levitated Materials. In Experimental Methods in the Physical Sciences; Elsevier, 2017; Vol. 49, pp 583-636. 
(34) Verbeni, R.; Pylkkänen, T.; Huotari, S.; Simonelli, L.; Vankó, G.; Martel, K.; Henriquet, C.; Monaco, G. Multiple-Element Spectrometer for Non-Resonant Inelastic X-Ray Spectroscopy of Electronic Excitations. J. Synchrotron Radiat. 2009, 16 (4), 469-476.

(35) Ponchut, C.; Rigal, J. M.; Clément, J.; Papillon, E.; Homs, A.; Petitdemange, S. MAXIPIX, a Fast Readout Photon-Counting X-Ray Area Detector for Synchrotron Applications. J. Instrum. 2011, 6 (01), C01069-C01069.

(36) Rueff, J.-P.; Ablett, J. M.; Céolin, D.; Prieur, D.; Moreno, Th.; Balédent, V.; Lassalle-Kaiser, B.; Rault, J. E.; Simon, M.; Shukla, A. The GALAXIES Beamline at the SOLEIL Synchrotron: Inelastic X-Ray Scattering and Photoelectron Spectroscopy in the Hard X-Ray Range. J. Synchrotron Radiat. 2015, 22 (1), 175-179.

(37) Ablett, J. M.; Prieur, D.; Céolin, D.; Lassalle-Kaiser, B.; Lebert, B.; Sauvage, M.; Moreno, Th.; Bac, S.; Balédent, V.; Ovono, A.; Morand, M.; Gélebart, F.; Shukla, A.; Rueff, J.-P. The GALAXIES Inelastic Hard X-Ray Scattering End-Station at Synchrotron SOLEIL. J. Synchrotron Radiat. 2019, 26 (1), 263 271.

(38) Lelong, G.; Radtke, G.; Cormier, L.; Bricha, H.; Rueff, J.-P.; Ablett, J. M.; Cabaret, D.; Gélébart, F.; Shukla, A. Detecting Non-Bridging Oxygens: Non-Resonant Inelastic X-Ray Scattering in Crystalline Lithium Borates. Inorg. Chem. 2014, 53, 10903-10908.

(39) Lee, S. K.; Eng, P. J.; Mao, H.-K.; Meng, Y.; Shu, J. Structure of Alkali Borate Glasses at High Pressure: B and Li K-Edge Inelastic X-Ray Scattering Study. Phys. Rev. Lett. 2007, 98, 105502.

(40) Fleet, M. E.; Muthupari, S. Boron K-Edge XANES of Borate and Borosilicate Minerals. Am. Mineral. 2000, 85, 11009-1021.

(41) Shaw, R. R.; Uhlmann, D. R. Subliquidus Immiscibility in Binary Alkali Borates. J. Am. Ceram. Soc. 1968, 51 (7), 377-382.

(42) Manuel, D.; Cabaret, D.; Brouder, Ch.; Sainctavit, Ph.; Bordage, A.; Trcera, N. Experimental Evidence of Thermal Fluctuations on the X-Ray Absorption near-Edge Structure at the Aluminum K Edge. Phys. Rev. B 2012, 85 (22), 224108.

(43) Yano, T.; Kunimine, N.; Shibata, S.; Yamane, M. Structural Investigation of Sodium Borate Glasses and Melts by Raman Spectroscopy. II. Conversion between $\mathrm{BO}_{4}$ and $\mathrm{BO}_{2} \mathrm{O}^{-}$Units at High Temperature. $J$. Non-Cryst. Solids 2003, 321, 9, 147-156.

(44) Wan, S.; Tang, X.; Sun, Y.; Zhang, G.; You, J.; Fu, P. Raman Spectroscopy and Density Functional Theory Analyses of the Melt Structure in $\mathrm{Li}_{2} \mathrm{~B}_{4} \mathrm{O}_{7}$ Crystal Growth System. Cryst. Eng. Comm. 2014, 16, 3086-3090.

(45) Alderman, O. L. G. Borate Melt Structure: A Short Review. Eur. J. Glass Sci. Technol. 2018, 59, 1-10.

(46) Varsamis, C.-P. E.; Vegiri, A.; Kamitsos, A. I. Molecular Dynamics Investigation of Lithium Borate Glasses: Local Structure and Ion Dynamics. Phys. Rev. B 2002, 65 (14), 104203.

(47) Zheng, G.; Yao, Y.; Zhang, S.; Wan, S.; You, J. Structural Studies of a $\mathrm{Li}_{2} \mathrm{O} \cdot 4 \mathrm{~B}_{2} \mathrm{O}_{3}$ Melt by HighTemperature Raman Spectroscopy and Density Functional Theory. CrystEngComm 2017, 19 (38), 57215726.

(48) Wright, A. C.; Vedishcheva, N. M. Borate Networks: Rigidity versus Dimensionality. Phys. Chem. Glas. Eur. J. Glass Sci. Technol. B 2016, 57 (1), 1-14.

(49) Kim, S. S.; Sanders, T. H. Calculation of Subliquidus Miscibility Gaps in the $\mathrm{Li}_{2} \mathrm{O}-\mathrm{B}_{2} \mathrm{O}_{3}-\mathrm{SiO}_{2}$ System. Ceram. Int. 2000, 26 (7), 769-778.

(50) Shartsis, L.; Capps, W.; Spinner, S. Density and Expansivity of Alkali Borates and Density Characteristics of Some Other Binary Glasses. J. Am. Ceram. Soc. 1953, 36 (2), 35-43.

(51) Wu, L.; Koryttseva, A.; Groß, C. B. M.; Navrotsky, A. Thermochemical Investigation of Lithium Borate Glasses and Crystals. J. Am. Ceram. Soc. 2019, 102 (8), 4538-4545.

(52) Ohkubo, T.; Gobet, M.; Sarou-Kanian, V.; Bessada, C.; Nozawa, M.; Iwadate, Y. Self-Diffusion Coefficient of Lithium in Molten $\mathrm{xLi}_{2} \mathrm{O}-(1-\mathrm{x}) \mathrm{B}_{2} \mathrm{O}_{3}$ System Using High-Temperature PFG NMR. Chem. Phys. Lett. 2012, 530, 4.

(53) Shi, X. M.; Wang, Q.; Li, C. X.; Niu, X. J.; Wang, F. P.; Lu, K. Q. Densities of $\mathrm{Li}_{2} \mathrm{O}-\mathrm{B}_{2} \mathrm{O}_{3}$ Melts. J. Cryst. Growth 2006, 290, 637-641.

(54) Montouillout, V.; Fan, H.; del Campo, L.; Ory, S.; Rakhmatullin, A.; Fayon, F.; Malki, M. Ionic Conductivity of Lithium Borate Glasses and Local Structure Probed by High Resolution Solid-Sate NMR. J. Non-Cryst. Solids 2018, 484, 57-64. 


\section{FOR TABLE OF CONTENT ONLY}
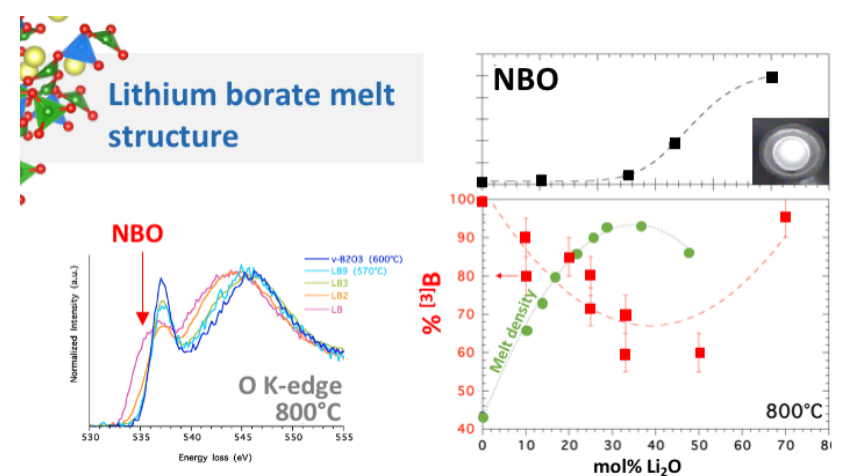

High-temperature X-ray Raman Spectroscopy has been used to determine the melt structure of five compositions of the $\mathrm{Li}_{2} \mathrm{O}-\mathrm{B}_{2} \mathrm{O}_{3}$ system. A temperature induced ${ }^{[4]} \mathrm{B}$ to ${ }^{[3]} \mathrm{B}$ conversion is observed above the glass transition temperature, and two distinct melt structures were evidenced for compositions below and above the diborate composition. These two structurally distinct melts allow explaining the two dynamical regimes observed for lithium ion diffusion. 